Ann. Zootech., I970, 19 (2), 235-237.

\title{
NOTE SUR UNE NOUVELLE MÉTHODE D'ESTIMATION DE LA CONFORMATION DES ANIMAUX
}

\author{
B.-L. DUMONT, P. LEGRAS * et J.-C. VERGÉ** \\ Laboratoire de Recherches sur la Viande, \\ ** Station centrale de physiologie animale \\ Centre national de Recherches zootechniques, 78 -Jouy-en-Josas \\ Institut national de la Recherche agronomique \\ * Institut technique de l'Élevage ovin et caprin, \\ 36 , rue Fontaine, 75 - Paris $(9 \mathrm{e})$
}

\section{RÉSUMÉ}

Un appareil destiné à relever et à enregistrer le profil corporel des animaux est décrit. Son principe consiste à mesurer le déplacement de tiges métalliques parallèles juxtaposées dans un support rigide, graduées et reliées à un système d'enregistrement électrique et mécanique mis en ouvre lors de l'application par pression de l'extrémité des tiges à différents niveaux de la surface corporelle.

La conformation des animaux de boucherie est l'un des éléments essentiels de leur valeur commerciale (Dumont et Arnoux, I968; Legras, 1970). Dans la pratique, le jugement de la conformation se réalise essentiellement par un examen subjectif du profil corporel à différents niveaux anatomiques considérés comme particulièrement importants au point de vue économique. Utilisée de longue date de façon empirique par les professionnels, cette méthode d'appréciation a été codifiée chez les bovins par la proposition d'un certain nombre de classes de profils musculaires (Houdinière, I957; WENIGER et al., 1966), et a servi à l'élaboration de tables de pointage utilisables notamment à des fins de contrôle des performances (Dumont et Duplan, 1959) ainsi qu'à l'étude ou à la définition des classes commerciales d'animaux sur les différents marchés français (Dumont, I967).

L'objectivation du jugement de la conformation à partir des profils musculaires est une nécessité en raison de la généralisation de l'emploi de ce système, en même temps que des difficultés liées à sa répétabilité.

Jusqu'à maintenant ce problème a été peu étudié, hormis par des méthodes de photogrammétrie, dont le coût et la complexité de l'appareillage limitent considérablement les possibilités d'emploi, comme en témoigne la rareté des publications dans ce domaine.

C'est pourquoi il nous a paru nécessaire dans le cadre d'une action concertée entre l'I. N. R. A. 
et l'I. T. O. V. I. C. sur la description des carcasses d'ovins, d'envisager un nouveau système d'appréciation de la conformation qui consiste, à l'aide d'un appareil approprié, à mesurer directement le profil des animaux (vivants ou en carcasse) à des niveaux déterminés.

L'appareil que nous proposons d'appeler un "profilmètre " consiste (cf. fig. I) en une série d'éléments métalliques mobiles numérotés et gradués, enchâssés dans un support transversal prenant appui sur deux barres rigides et fixes qui limitent latéralement le champ de mesure. Ces barres et le support transversal font partie du corps de l'appareil qui comprend, protégés par un carter, les différents systèmes d'enregistrement. Des poignées situées de part et d'autre du corps de l'appareil permettent de maintenir ce dernier dans la position souhaitée par rapport aux profils à mesurer dont on peut noter la situation grâce à des niveaux intégrés à l'appareil.

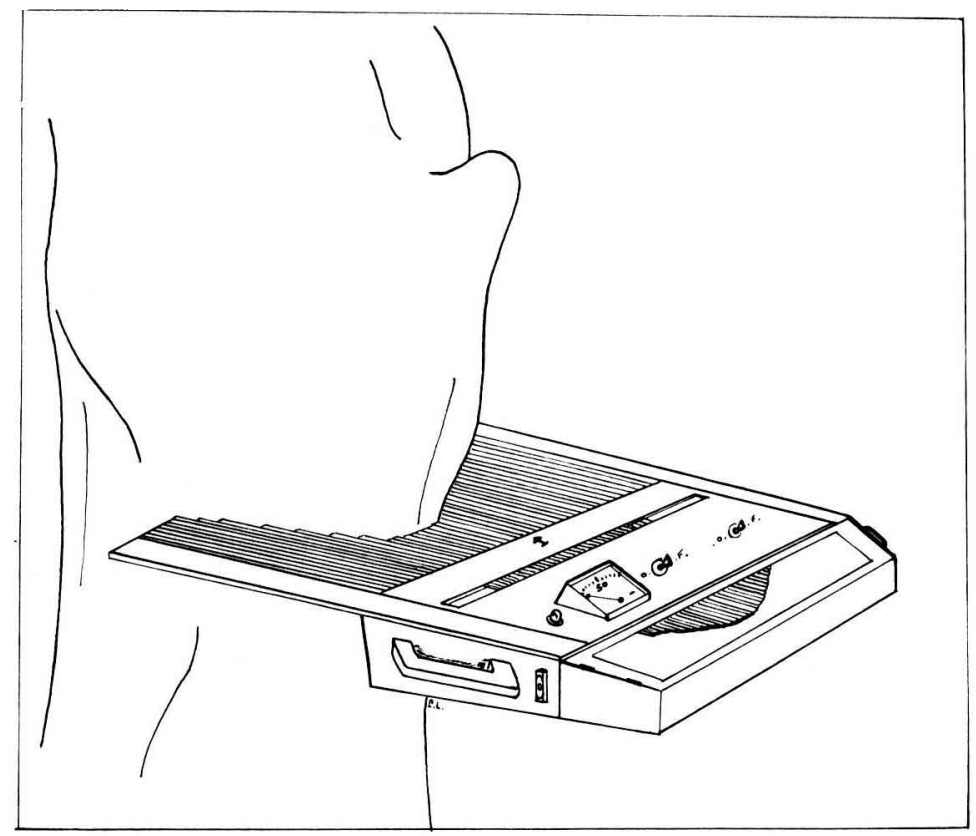

FIG. I. - Schéma du "Profilmètre " en utilisation sur une carcasse d'ovin

Pour réaliser la mesure on applique les éléments métalliques contre la surface dont on veut contrôler le profil en poussant, grâce aux poignées, l'appareil dans la direction souhaitée jusqu'à ce que les extrémités des éléments aient complètement épousé le profil. Un dispositif de serrage permet alors de bloquer les éléments dans leur position.

L'enregistrement du déplacement des éléments métalliques peut donner lieu à plusieurs sortes de mesures.

Individuellement on peut déterminer l'enfoncement de chacun d'eux par lecture de sa graduation au niveau de la barre transversale qui le supporte. On peut aussi envisager de traduire cet enfoncement par la mesure de la résistance électrique de la portion enfoncée dans le corps de l'appareil.

Par un dispositif approprié de relais cette mesure peut être transmise à un système enregistreur extérieur à l'appareil et utilisée dans tout calcul souhaitable.

Globalement on peut retenir la mesure de la somme des résistances de la portion enfoncée des différents éléments pour traduire le "rebondi " du profil. Cette dernière mesure peut être réalisée grâce à un ohmmètre placé sur le corps de l'appareil et alimenté par piles. On peut aussi obtenir 
un relevé en vraie grandeur du profil, mesuré par enregistrement sur papier du positionnement de l'extrémité des éléments métalliques, dans leur partie située dans le corps de l'appareil.

L'appareil dont nous envisageons de réaliser plusieurs modèles commerciaux de largeur variable, adaptée au relevé de profils dans différentes espèces est d'un emploi commode.

Reçu pour publication en mai 1970.

\section{SUMMARY}

\section{AN APPARATUS FOR RECORDING CONFORMATION}

An apparatus for recording conformation called a "profilemeter " is described. The disp'acement of parallel metallic spindles, placed side by side in a rigid support, is measured. The spindles are graduated and connected to an electrical and mechanical recording system which functions by pressing the point of the spindles upon the body surface at different levels.

\section{RÉFÉRENCES BIBLIOGRAPHIQUES}

Dumont B.-L., Duplan J. M., r959. Jugement des animaux vivants sur leurs performances ; crojs. sance, précocité, conformation. Établissement d'une table de pointage. Agriculture, 214, 207-212.

Dumont B.-L., I967. Étude sur les qualités des carcasses de bovins en France. Informations statistiques $\mathrm{n}^{\circ}, 4$.

Dumont B.-L., Arnoux J., rg68. Influence de différents caractères de la carcasse de bovins sur la détermination de son prix. Etude de statistique agricole $\mathrm{I}$.

Houdinière A., I957. L'examen des " profils musculaires n dans l'appréciation de la qualité des viandes. Bull. Acad. Vet., 30, 1, 51-62.

Legras P., I970. Ce qu'il faut connaître sur les carcasses et la production de viande chez les ovins. S. P. E. O. Pâtre Édit. Paris.

Weniger J.-H., Dumont B.-L., de Boer H., Bergstrom P.-L., Engelke F., Glodek P., I966. Étude sur les qualités de carcasses de bovins et porcins dans les pays de la Communauté Économique Européenne. Office Statistique des Communautés Européennes. Bruxelles.

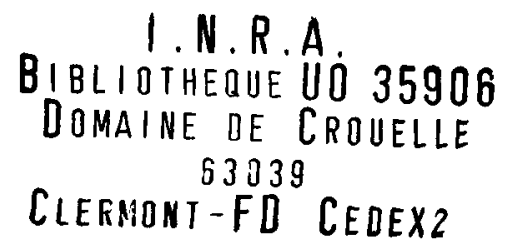

